\title{
Scattering of biflagellate microswimmers from surfaces
}

\author{
Enkeleida Lushi, ${ }^{1, *}$ Vasily Kantsler, ${ }^{2, \dagger}$ and Raymond E. Goldstein ${ }^{3, \dagger}$ \\ ${ }^{1}$ Courant Institute of Mathematical Sciences, New York University, New York 10003, USA \\ ${ }^{2}$ Department of Physics, University of Warwick, Coventry CV4 7AL, United Kingdom \\ ${ }^{3}$ Department of Applied Mathematics and Theoretical Physics, University of Cambridge, Cambridge CB3 OWA, United Kingdom
}

(Received 8 August 2016; revised manuscript received 19 July 2017; published 10 August 2017)

\begin{abstract}
We use a three-bead-spring model to investigate the dynamics of biflagellate microswimmers near a surface. While the primary dynamics and scattering are governed by geometric-dependent direct contact, the fluid flows generated by the swimmer locomotion are important in orienting it toward or away from the surface. Flagellar noise and in particular cell spinning about the main axis help a surface-trapped swimmer escape, whereas the time a swimmer spends at the surface depends on the incident angle. The dynamics results from a nuanced interplay of direct collisions, hydrodynamics, noise, and the swimmer geometry. We show that to correctly capture the dynamics of a biflagellate swimmer, minimal models need to resolve the shape asymmetry.
\end{abstract}

DOI: 10.1103/PhysRevE.96.023102

\section{INTRODUCTION}

Microscopic swimming cells such as bacteria, algae, and spermatozoa live in porous natural habitats and this geometrical confinement necessitates interactions with surfaces. Surface interactions are fundamental in many biological processes, e.g., biofilm formation and egg fertilization; however, despite their ubiquity in nature, not all is known about the physics of microlocomotion in a confined environment [1]. Better understanding of the dynamics of micro-organisms near surfaces is crucial for many applications to control microbial locomotion [2].

There has been considerable discussion of whether Brownian and intrinsic noise, long-range hydrodynamic or shortrange mechanical forces determine the surface interactions of these micro-organisms [1,3-11]. Microswimmers such as bacteria or spermatozoa accumulate near boundaries $[1,12]$ and navigate alongside them [13-15]. Hydrodynamic and steric interactions with surfaces are credited for the circling behavior of individual bacteria in surfaces [16-18], the boundary following of artificial motile colloids [19-21], and the emergence of collective motion in confined bacterial suspensions [22-25]. Direct measurements of the flow fields around an individual bacterium reveal that the intrinsic stochasticity of its motion drowns the effects of long-range fluid dynamics and implies that surface interactions of bacteria are dominated by the direct collisions and Brownian noise $[4,26]$. Eukaryotic swimmers such as algae $C$. reinhardtii scatter off a surface by pushing against it with their flagella $[7,10]$, indicating that the primary surface dynamics is governed by direct or steric interactions. For $C$. reinhardtii it was shown that the swimmer geometry and flagellum length are crucial in determining the surface scattering [7].

\footnotetext{
*lushi@cims.nyu.edu

†V.Kantsler@warwick.ac.uk

${ }^{\ddagger}$ R.E.Goldstein@damtp.cam.ac.uk
}

Published by the American Physical Society under the terms of the Creative Commons Attribution 4.0 International license. Further distribution of this work must maintain attribution to the author(s) and the published article's title, journal citation, and DOI.
Hydrodynamics however, while seemingly drowned out by the noise and obscured by the complex surface interaction, is ever present and affects the swimmer motion. Using a simple model biflagellate microswimmer, we investigate here the delicate interplay between the swimmer geometry, flagella, the generated fluid flows, and noise in its interaction with a surface. The swimmer, consisting of three beads connected by elastic springs (see Fig. 1), moves with constant propulsive flagellar forces and generates a "puller" disturbance fluid flow resembling the experimental observations $[26,27]$. We show that while the primary scattering dynamics is indeed governed by the swimmer geometry and steric effects, the attraction or repulsion of the swimmer to surfaces is influenced by fluid dynamics. The scattering angle is shown to depend on the swimmer geometry, as also observed in experiments [7]. We show that noise and in particular cell spinning help surface-trapped swimmers escape. The time that a puller biflagellate swimmer spends at a surface is shown to depend quasilinearly on the incoming angle, a result confirmed with our experimental measurements.

We also establish here the appropriate levels of simplicity in microswimmer models so that they capture the behavior of biflagellates as is observed in experiments with $C$. reinhardtii. Since the dynamics near a wall depends primarily on direct contact of the flagella, we show that an asymmetric or triangular shape is needed to capture the scattering phenomenon. Moreover, since such swimmers spin about their axis and scattering depends on the cell configuration [10], we show that three-bead models are the most minimal that can still display the correct physical behavior.

\section{MODEL}

We consider a minimal three-bead-spring model for $C$. reinhardtii as illustrated in Fig. 1(a). The model is inspired by the recent experimental measurements of the flow field around this type of algae $[26,27]$, which can be approximated reasonably well by three Stokeslets. The three-Stokeslet description has been recently used to investigate phenomena such as the coupled flagellum dynamics and synchronization [28-30], tumbling [31], and phototaxis [32,33] in C. reinhardtii. 

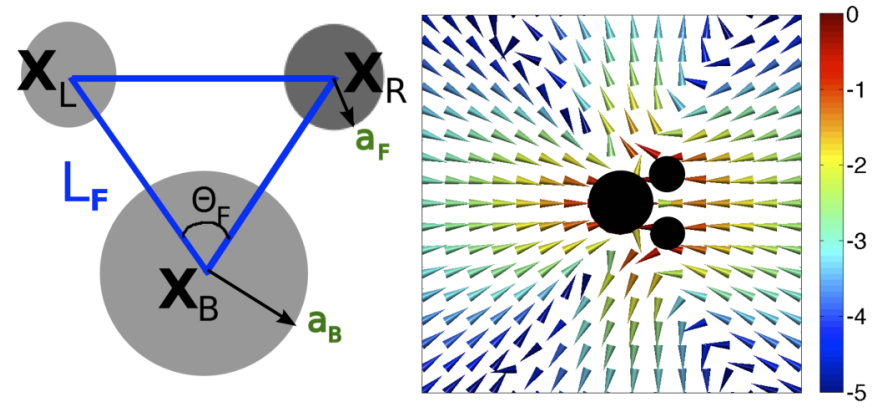

FIG. 1. Shown on the left is a diagram of the three-bead swimmer and on the right is the disturbance fluid flow in free space; the color represents flow magnitude on a logarithmic scale.

We assume that constant propulsive forces are concentrated on the flagellum beads with radius half the body bead. Using a zero force condition on the entire swimmer and a balance of forces on each bead (similar to the two-bead puller model of Hernandez-Ortiz et al. [34,35]), we can derive the equation of motion for each bead. The coupled dynamics of the beads $B, L, R$ (denoting the swimmer body, left flagellum bead, and right flagellum bead, respectively) can be compactly described as

$$
\begin{aligned}
\frac{d \mathbf{x}_{k}}{d t}= & \frac{1}{\xi_{k}}\left[\delta_{k,(L, R)} \mathbf{f}_{k}^{f}+\mathbf{f}_{k}^{c}+\mathbf{f}_{k}^{x}\right] \\
& +\sum_{j=(B, L, R)} G_{a_{j}}\left(\mathbf{x}_{k}, \mathbf{x}_{j}\right)\left[\left(1-\delta_{k, j}\right) \mathbf{f}_{j}^{c}+\mathbf{f}_{j}^{x}\right] \\
& +\sum_{j=(B, L, R)} \tilde{G}_{a_{j}}\left(\mathbf{x}_{k}, \mathbf{x}_{j}\right)\left(\mathbf{f}_{j}^{c}+\mathbf{f}_{j}^{x}\right)
\end{aligned}
$$

for $k=(B, L, R)$. Here $\xi_{k}=6 \pi a_{k}$ are Stokes drag coefficients and $a_{B}=1 / 3$ and $a_{L}=a_{R}:=a_{F}=1 / 6$ are the bead radii. The propulsive forces $\mathbf{f}_{L}^{f}$ and $\mathbf{f}_{R}^{f}$ act only on the two flagellum beads $L$ and $R$. The connector spring forces $\mathbf{f}^{c}$ are calculated using the finitely extensible nonlinear elastic spring model introduced by Hernandez-Ortiz et al. in [35] for their twobead-spring swimmers. The spring constant is $h=25$ and the springs are finitely extensible with $L_{\max } / L=1.01$ and $L_{\min } / L=0.99$. The steric forces $\mathbf{f}_{k}^{x}$ are calculated with the short-range and purely repulsive Weeks-Chandler-Anderson potential [36] activated at a distance of $2^{1 / 6} a \approx 1.12 a$ from a bead's center to prevent overlaps. Other potentials can be used as well, but they should not be long range. In Eq. (1)

$$
G_{a}\left(\mathbf{x}_{k}, \mathbf{x}_{j}\right)=\frac{1}{8 \pi}\left[\frac{r^{2}+2 a^{2}}{\left(r^{2}+a^{2}\right)^{3 / 2}} \mathbf{I}+\frac{\left(\mathbf{x}_{k}-\mathbf{x}_{j}\right)\left(\mathbf{x}_{k}-\mathbf{x}_{j}\right)^{T}}{\left(r^{2}+a^{2}\right)^{3 / 2}}\right],
$$

with $r=\left\|\mathbf{x}_{k}-\mathbf{x}_{j}\right\|$ is a regularized Stokeslet in three dimensions where the regularization parameter $a$ is the bead radius [37]. If the swimmer is near a no-slip wall, the method of images with regularized image Stokeslets [38], here denoted by $\tilde{G}_{a}$, is employed. The fluid velocity at some point $\mathbf{x}_{e}$ is

$$
\mathbf{u}\left(\mathbf{x}_{e}\right)=\sum_{j=(B, L, R)}\left[G_{a_{j}}\left(\mathbf{x}_{e}, \mathbf{x}_{j}\right)+\tilde{G}_{a_{j}}\left(\mathbf{x}_{e}, \mathbf{x}_{j}\right)\right]\left(\mathbf{f}_{j}^{c}+\mathbf{f}_{j}^{x}\right) .
$$

If steric forces, necessary in problems involving confinement, are included in the model, then the Stokeslets need not be regularized. However, for fast simulations of many such swimmers in the bulk, the regularized Stokeslet is an easy way to avoid there costly computations of steric interactions while still retaining some information about the swimmer size. We include them in results shown here.

The wall is at $z=0$ and the swimmer in the $z>0$ half space. The dynamics is in three dimensions, but, in the absence of body and flagellum rotation, the swimmer motion remains planar and with direction $\mathbf{n}=\left(\mathbf{x}_{L}+\mathbf{x}_{R}\right) / 2-\mathbf{x}_{B}$ for a puller swimmer. Propulsive flagellum forces $\mathbf{f}_{L}^{f}$ and $\mathbf{f}_{R}^{f}$ [cf. Fig. 1(a)] are taken to be $-\mathbf{n} /|\mathbf{n}| / 2$ and fixed in magnitude.

For a "pusher" swimmer, like the moving-backward-only mutant Chlamydomonas CC-2679 mbo1 [7], we reverse the direction of the applied flagellar forces $\mathbf{f}^{f}$ [see Fig. 1(a)]. The swimmer then moves head-bead first with direction $\mathbf{n}=$ $\mathbf{x}_{B}-\left(\mathbf{x}_{L}+\mathbf{x}_{R}\right) / 2$. The generated flow field generated then is pusherlike and with the direction reversed from the puller case in Fig. 1(b).

\section{MATERIALS AND METHODS}

The experiments' protocol followed here is exactly that followed in the forerunning study of Kantsler et al. [7]. C. reinhardtii strains CC-125 wild type (WT), CC-2347 shf1-277, CC-2289 lf3-2, and CC-2679 mbo1 (The Chlamydomonas Resource Center) were grown and used. Quasi-two-dimensional microfluidic channels were manufactured with standard soft lithography techniques. Swimming characteristics of individual algae cells and their trajectories were reconstructed by applying a custom-made particle tracking-velocimetry algorithm to image data taken with a Nikon TE2000-U inverted microscope [10 $\times$ objective, 10 frames/s (fps)]. The flagellum dynamics close to the boundary were captured with a Fastcam SA-3 Photron camera (500-2000 fps, 40×/numerical aperture (NA) 1.3 oil immersion and $60 \times / \mathrm{NA} 1.0$ water immersion objectives).

\section{FLUID DYNAMICS}

Figure 2 illustrates the swimmer dynamics near a wall. Pusher and puller swimmers behave differently: Puller swimmers scatter off the wall as in Fig. 2(a), which resembles experimental observations in Fig. 2(b), whereas pushers tend to swim alongside the wall, as in Fig. 2(d). Surprisingly, this behavior partly depends on the generated fluid flows. If the beads interact only through the springs and the hydrodynamics is neglected, the puller swimmer remains at the wall and does not scatter [Fig. 2(a)]. Without hydrodynamics, a pusher swimmer does not notice the presence of a corner in Fig. 2(d). In experiments, a spermatozoon, which is a pusher, is observed to swim past a corner, making a negative angle to the horizontal [7]. Our model captures this phenomenon, as shown in Fig. 2(d), and demonstrates that it results from the hydrodynamic attraction to the vertical side of the corner. The corner is rounded with a radius similar to the flagellum beads for ease of computation and interactions are computed accordingly. The importance of fluid dynamics is obvious in the swimmer orientation as it approaches the boundary: The 


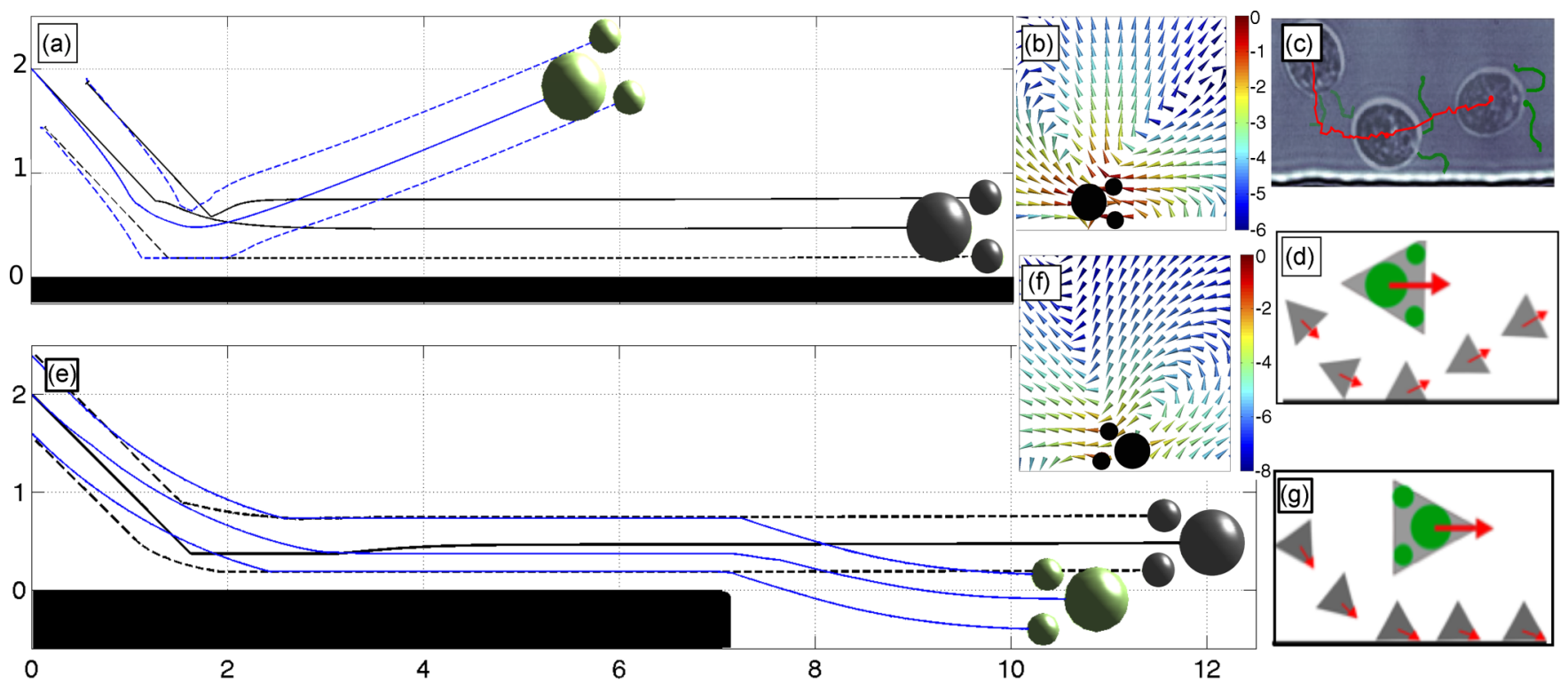

FIG. 2. (a) Trajectories of a puller swimmer with and without hydrodynamics [the beads are shown in green (gray) and their trajectories in blue (black)]. (b) Fluid flow generated when the puller swimmer hits the wall. (c) Scattering trajectory of a wild-type $C$. reinhardtii [7]. (e) Trajectories of a pusher swimmer with and without hydrodynamics [beads in green (gray) and their trajectories in blue (black)], along the wall and past a smoothed $90^{\circ}$ corner. (f) Fluid flow generated by the pusher swimmer at the wall. (d) and (g) Swimmer triangular geometry scattering argument suggested by Kantsler et al. [7]. See the Supplemental Material [39] for movies of the dynamics.

pusher in Fig. 2(d) tends to align parallel with the wall, whereas the puller in Fig. 2(a) tends to orient perpendicular to it.

\section{SCATTERING}

In Fig. 2(a) we show a typical trajectory of a threebead puller swimmer when the incoming angle $\theta_{i n}$ before the collision is not steep $\left(\theta_{i n}+\theta_{F} / 2<\pi / 2\right)$. At the wall the swimmer executes a turn. The lower flagellum bead experiences more drag [as can be seen by the asymmetry of the configuration in Figs. 3(b) and 3(c)] and slides along the wall. Spring forces push on the other flagellum and body bead seeking to return to the rest configuration. This results in the swimmer turning at the wall in a finite time and, once reoriented, swimming away from the wall.

We measure the scattering angle $\theta_{\text {out }}$ for a variety of incidence angles $\theta_{i n}$ and swimmer flagellum lengths $L_{F}$ but keeping fixed the interflagellum angle $\theta_{F}=70^{\circ}$. The results are shown in Fig. 3(a). Angles are measured at a distance $1.2 a_{F}$ above the wall and are with respect to the horizontal surface, while "touching" of the wall is implied when the steric forces are activated. (Changing the criterion for measuring the incoming and outgoing angles changes the results only qualitatively and minimally.) We notice in Fig. 3(a) that the angle at which a swimmer scatters of a wall does not seem to depend on the incidence angle, with the angles differing by less than $5^{\circ}$. This suggests that the memory of the incoming angle is lost during the swimmer turning at the wall, especially for steep incident angles.

For completeness, we plot in Fig. 3(b) the scattering angle vs the incident angle for four different strains of Chlamydomonas: the WT CC-125 with flagellum length 11-13 $\mu \mathrm{m}$, the short-flagellum mutant CC- 2347 shf1 with flagellum length 6-8 $\mu \mathrm{m}$, the long-flagellum mutant CC-2289 lf3-2 with flagellum length $12-22 \mu \mathrm{m}$, and the moving-backward-only mutant CC-2679 mbol, which is considered a pusher swimmer just like bacteria [7]. The trajectory angles are measured at a distance $20 \mu \mathrm{m}$ from the wall. In the experiments the scattering angle of the puller swimmer does not differ much with the incident angle, but increases with the flagellum length. The scattering angle for the pusher swimmer is very low $\left(5^{\circ}\right)$, indicating that it swims along the wall just like bacteria.

The scattering angle in the model here does not vary much with the flagellum length, as can be seen in Fig. 3(a), whereas in the experiments the scattering angle increases with the flagellum length, as can be seen in Fig. 3(b) and analyzed in [7]. Since the flagella in $C$. reinhardtii are not straight but are flexible, their change in length is also a change in the swimmer triangular geometry. With the model we can investigate the importance of the geometric shape and direct ciliary contact with the wall by varying the interflagellum angle $\theta_{F}$ but fixing the flagellum length $L_{B}=4 a$ and incidence angle $\theta_{i n}=45^{\circ}$. Figure 3(c) shows a pronounced increase of the scattering angle with increasing $\theta_{F}$, confirming the argument that scattering depends mostly on the swimmer triangular geometry just as proposed by Kantsler et al. [7] and illustrated in Figs. 2(f) and $2(\mathrm{~g})$.

\section{IMPACT TIME}

We discuss the time it takes a puller swimmer to turn at the wall, or impact time, which is measured as the time during which a flagellum touches the wall. We observe three distinct states. (i) One is hydrodynamic attraction of the swimmer to the wall when starting with a quasihorizontal configuration, as illustrated in Fig. 3(d), and then escape from the wall without 
(a)

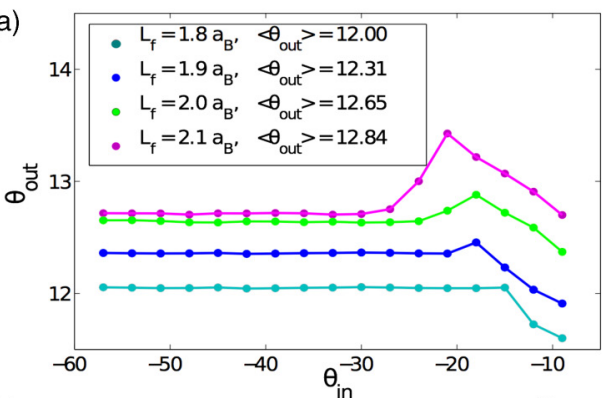

(d)

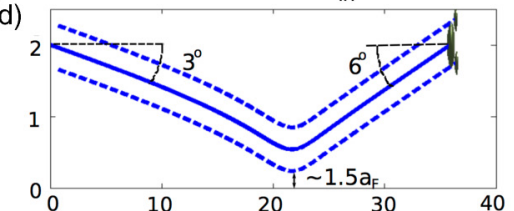

(e)

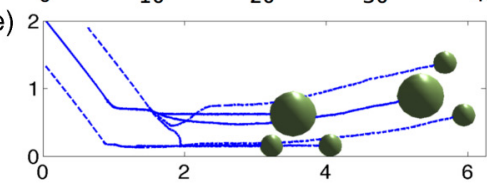

(b)
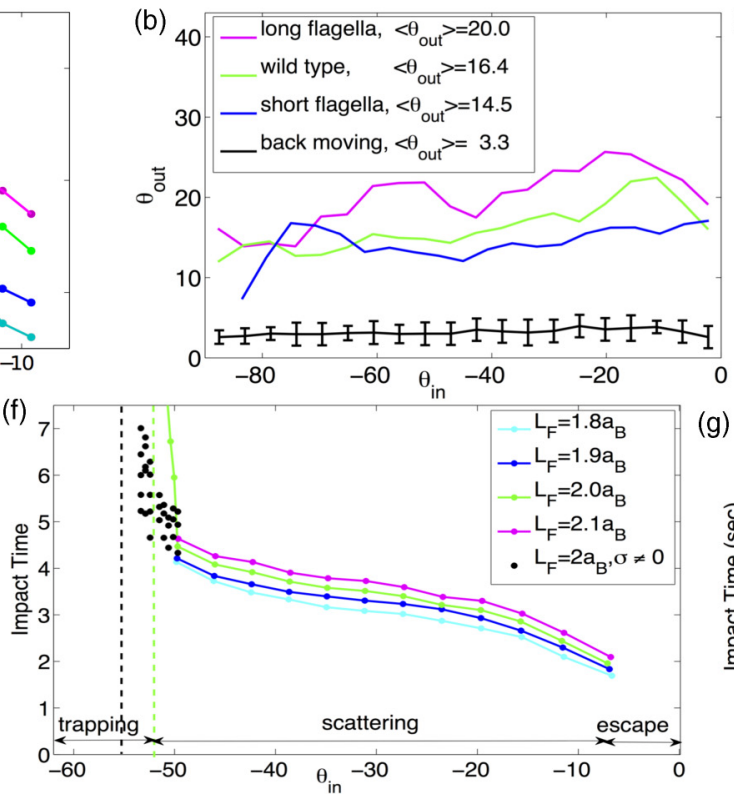

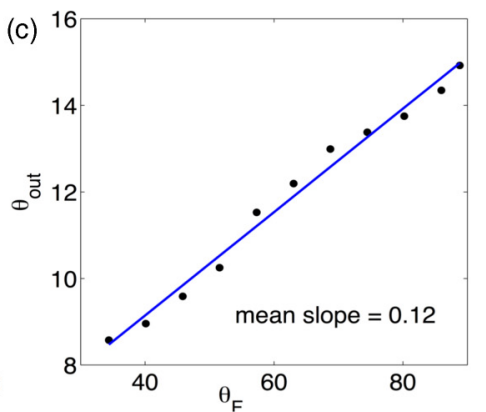

(g)

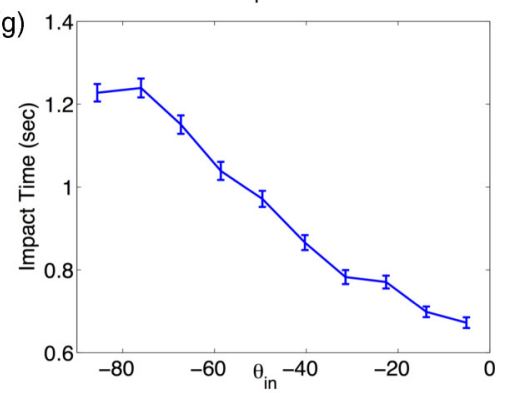

FIG. 3. (a) Scattering angles of a puller swimmer vs incident angles for four different swimmer flagellum length and fixed interflagellum angle $\theta_{F}=70^{\circ}$. (b) Experimentally measured scattering angles vs incident angles for four types of $C$. reinhardtii: three puller types with different flagellum lengths and one pusher type. (c) Scattering angle for a variety of interflagellum angle $\theta_{F}=70^{\circ}$ keeping fixed the incidence angle $\theta_{i n}=45^{\circ}$ and flagellum length $L_{F}=2 a_{B}$. (d) Swimmer hydrodynamically interacting with the wall without touching it. (e) Swimmer is trapped at the wall, but it can scatter if noise is included in the dynamics. (f) Impact time of a puller swimmer at the wall vs incident angles for different flagellum lengths and with noise. (g) Experimentally measured impact time for wild-type C. reinhardtii. Angles are reported in degrees.

touching it. The angle of escape is different from the angle of approach, indicating the effect of the asymmetrical placement of the Stokeslets. The impact time in this regime is exactly zero. (ii) Another is an intermediate regime of scattering dynamics where the dependence of the impact (turning) time to the incident angle is almost linear; the swimmers approaching with a steep angle take more time to turn at the wall. Figure 3(f) shows this impact time for a variety of incident angles and flagellum lengths but fixed interflagellum angle $\theta_{F}=70^{\circ}$. (iii) The third is a regime where the swimmer gets trapped at the surface with the flagella touching the wall, as can be seen in Fig. 1(e). This typically happens for $\theta_{i n}>\left(\pi-\theta_{F}\right) / 2$. The impact time is infinite, thus there is a vertical asymptote, as shown in Fig. 3(f).

We measured the impact time for WT Chlamydomonas for a variety of incident angles, as shown in Fig. 3(g). If the incident angles not very steep, the impact time also grows quasilinearly. However, the impact time tapers off for high incident angles, indicating that the swimmers in the experiments do not become trapped.

\section{NOISE}

It has been shown that in $C$. reinhardtii the flagellum pair apparent asynchronization and slips result in randomization of the micro-organism motion [40]. Flagella proximity to a surface might also significantly increase the pair asynchronization probability. We can incorporate this intrinsic noise in our model by including noise with strength $\sigma$ in the flagellum bead dynamics as $d \mathbf{x}_{k} / d t=\left(d \mathbf{x}_{k} / d t\right)_{\text {deterministic }}+\xi_{k}(t)$, where $\xi_{k}(t)$ has a Gaussian probability distribution with zero mean and correlation function $\left\langle\xi_{k}(t), \xi_{j}\left(t^{\prime}\right)\right\rangle \sigma^{2} \delta_{k j} \delta\left(t-t^{\prime}\right)$.

We illustrate how sufficient noise can help a swimmer overcome surface trapping. In Fig. 3(e) we show an example where noise added to the flagellar dynamics enables escape, whereas the same initial condition for a noiseless swimmer results in it being bound to the surface. Note that the position of the vertical asymptote in the impact time vs incident angle plot shown in Fig. 3(f) is pushed further to the left for examples with flagellar noise, illustrating how sufficient noise can help in the marginal cases. For $C$. reinhardtii, however, there are other robust mechanisms that can help swimmers free themselves from surface entrapments [10], which we discuss later.

\section{ONE, TWO, OR THREE BEADS?}

We compare the dynamics and trajectories of bead-spring puller swimmers near a wall. The two-bead model has been used to study the collective behavior of front-back symmetric swimmers [34,35], i.e., the swimmer body and flagellum beads are of equal size. A symmetric two-bead puller, shown in Fig. 4(b), reaches the wall but due to hydrodynamic interaction with its own image, repels away from it while still oriented quasiparallel to the wall.

A big-tail swimmer where the front flagellum bead is smaller than the back body bead collides with the wall and moves along it, as in Fig. 4(a). It cannot turn away from the wall due to its shape asymmetry and hence is trapped. A big-head swimmer where the front flagellum bead radius is bigger broadly mimics the triangular geometry of $C$. reinhardtii [41]. Such a puller swimmer (with hydrodynamics accounted for) 

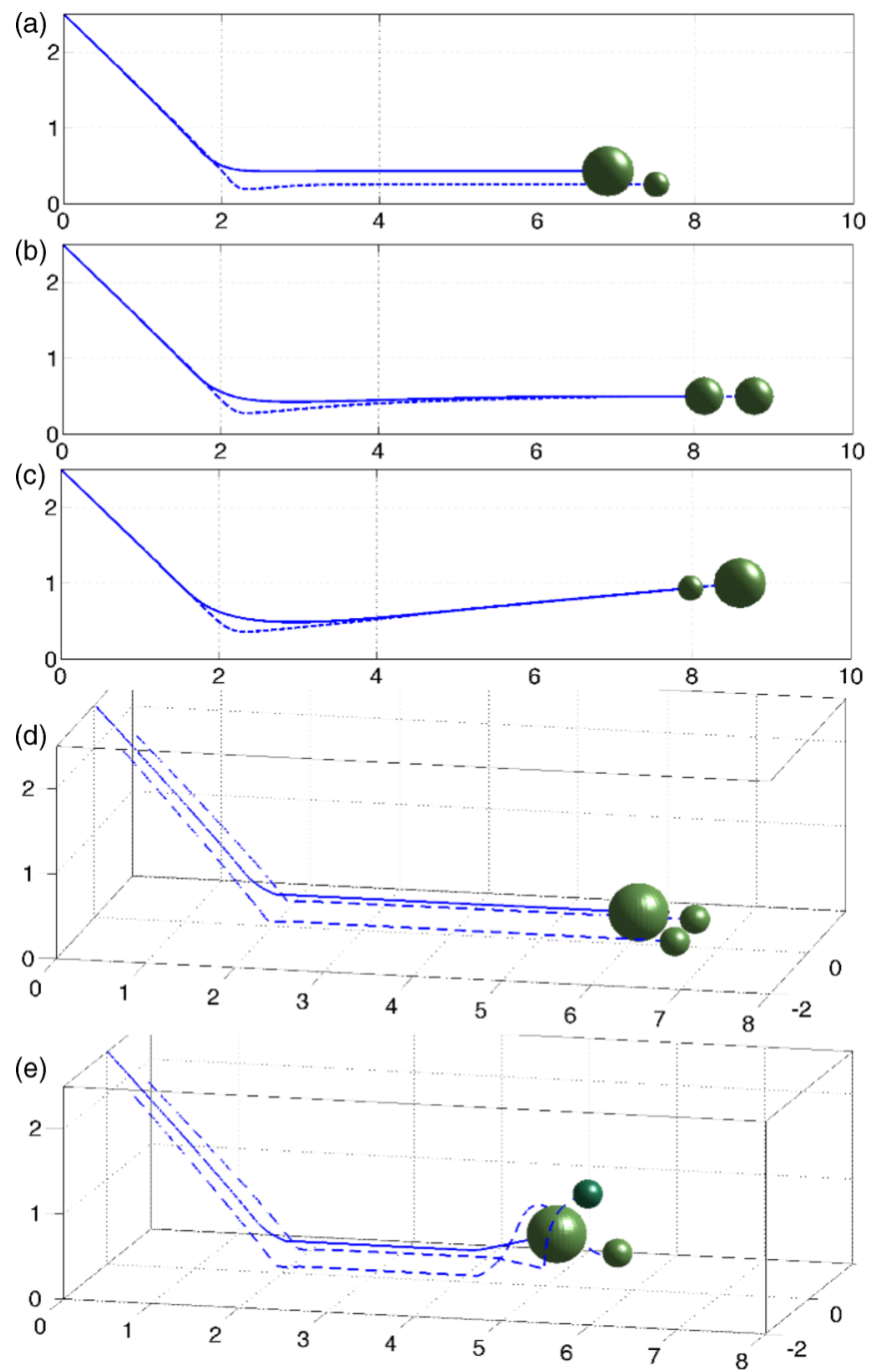

FIG. 4. Microswimmer dynamics near a wall: comparisons of trajectories obtained with different models. (a) Asymmetric big-tail two-bead-spring puller swimmer with the body bead twice the radius of the flagellum bead. (b) Symmetric two-bead-spring puller swimmer where the body and flagellum beads have the same radii. (c) Big-head two-bead-spring puller swimmer with the body bead half the radius of the flagellum bead. (d) Three-bead-spring puller swimmer as in Fig. 1 where the swimmer flagellum configuration is parallel to the surface gets trapped there. (e) Cell spinning enables the swimmer to escape the wall. See the Supplemental Material [39] for movies of the dynamics in case (e) and also another case of spinning-enabled escape.

can scatter away from the wall. The escape angle is determined by its geometry, as can be seen in Fig. 4(c). This confirms that an asymmetric swimmer shape is indeed needed to obtain scattering dynamics.

Big-head swimmers however are axisymmetric and do not adequately model the geometry of a biflagellate like $C$. reinhardtii whose generated fluid flows are better captured by three Stokeslets $[26,27]$. While both the big-head model and the three-bead model capture the scattering dynamics, the latter captures the dynamics of both flagella, which clearly do not remain symmetric when touching a wall.

\section{CELL CONFIGURATION AND SPINNING}

In the cases presented in Fig. 2, the plane of motion for the triangular swimmer is perpendicular to the wall plane. If the swimmer plane of motion is instead so that the flagellar plane is parallel to the wall [see Fig. 4(d)], the swimmer has two possible dynamics scenarios: (a) If the incoming angle is steep, then it gets trapped at the wall and does not scatter, or (b) if the incoming angle is quasiparallel to the wall, then it experiences a slight attraction at the wall due to hydrodynamics, but never touches it. The trapping case is conceptually similar to that of Fig. 4(a), where the swimmer cannot turn away from the wall, whereas the hydrodynamic attraction case is similar to that of Fig. 3(d). Note that in this configuration the swimmer does not have a scattering phase at all. Since $C$. reinhardtii has not been observed in experiments to get trapped at the wall [see Fig. 3(g)], the trajectory in Fig. 4(d) indicates that other mechanisms may prove important in resolving the dynamics at the wall $[10,42]$.

Comparing the simulations and experiments in Figs. 3(f) and $3(\mathrm{~g})$, we notice that $C$. reinhardtii spends a finite time at the wall and eventually escapes, whereas the model swimmer can get trapped there indefinitely. The discrepancies may result from the fact that Chlamydomonas slowly spins about its main axis and it was recently observed in experiments that this feature enables the swimmer to escape cylindrical obstacles with a diameter of about $25 \mu \mathrm{m}[10]$.

We can check the cell spinning effect with our model here by adding a slow rotation of the flagellar beads about the swimmer's main axis of motion, akin to the effective torque discussed in [43] to mimic flagellar activity. We test the spinning three-bead-spring model in the case of the trapped swimmer of Fig. 4(d) and turn on the rotation after the swimmer has spent some time moving alongside the wall. After the cell has rotated by about $90^{\circ}$, it is effectively in the configuration where the flagellar plane is perpendicular to the wall, which, as discussed before and illustrated in Figs. 2(a), 2(c), and 2(e), allows the swimmer to scatter off the wall due to its triangular shape. When the swimmer is in the trapped position shown in Fig. 3(e), cell spinning allows it to first get in the position shown in Fig. 4(d) and then scatter similarly to Fig. 4(e). The movies in the Supplemental Material [39] illustrate this effect: No matter the initial swimmer configuration, if the swimmer is "trapped" at the wall, cell spinning allows it to turn to a favorable position and scatter. Cell spinning thus enables biflagellate swimmers to escape from surfaces.

\section{DISCUSSION}

We introduced a model for microswimmers where the body and two flagella are represented by spheres connected by elastic springs. The dynamics of a biflagellate swimmers near straight surfaces was investigated and the roles of the direct collisions, fluid flows, and noise are discussed in detail. Some recent studies $[41,44]$ consider the swimmer shape asymmetry, e.g., big-head swimmers, but they do not include hydrodynamics. Others consider asymmetric hydrodynamics, but neglect asymmetric shape interactions $[45,46]$. Here we show that the 
dynamics of biflagellates near surfaces is a complex interplay of all these ingredients (shape, hydrodynamics, and noise) and the roles of each ingredient should not be neglected in models and computations involving swimmers near surfaces. In particular, our results show that the swimmer size and shape should be resolved to obtain the correct dynamics near surfaces. More simplified microswimmer models, such as a one-point dipole or a squirmer swimmer, may be inadequate.

Just as recent experiments suggest [7,10], we find that the scattering of puller swimmers like $C$. reinhardtii depends primarily on its triangular geometry and the direct flagellar contact with the surface and secondarily on the hydrodynamic interaction with the surface. While in the $C$. reinhardtii swimmer the scattering angle depends on the flagellum length, in the model three-bead-spring swimmer it depends on the interflagellum angle. Both these show that the triangular swimmer geometry is an important factor in determining the scattering angle. Noise is shown to help model swimmers escape surface entrapment, however it is the cell spinning about its main axis that enables the swimmer to escape from the wall. The time a puller swimmer spends to turn at the wall is shown to depend quasilinearly on the incoming angle, an observation confirmed by experiments with wild-type $C$. reinhardtii. This turning or detention time [43] at the wall is nonzero and nonconstant, indicating that point-billiard models of micro-organisms just reflecting at the wall are oversimplified and may miss this crucial dynamics. The ability to avoid long-term trapping at surfaces represents a significant advantage for a soil alga like Chlamydomonas, which in its natural habitat navigates a porous material [10].

The three-bead-spring model presented here is versatile and can be adapted to study interactions of biflagellates with more complex surfaces and a variety of confinements, e.g., circular chambers as in Ref. [47], by changing or approximating the form of the Stokeslets in Eq. (1) to the appropriate ones for the surfaces in question. A time-dependent breaststroke or a swimmer spinning about its main axis, as observed in the motion of the $C$. reinhardtii algae, can be incorporated to model the motion. Moreover, it is possible to include lubrication hydrodynamics to further clarify the motion of the spherical body near a surface [10]. Finally, this three-bead-spring model can be used to study the collective motion of many biflagellates in diverse geometrical confinements and heterogeneous porous media mimicking their natural habitats.

\section{ACKNOWLEDGMENTS}

We thank M. Contino, J. Dunkel, K. Leptos, M. Polin, and I. Tuval for many helpful discussions. This work was supported in part by an Established Career Fellowship from the Engineering and Physical Sciences Research Council (REG).
[1] A. P. Berke, L. Turner, H. C. Berg, and E. Lauga, Phys. Rev. Lett. 101, 038102 (2008).

[2] R. Rusconi, M. Garren, and R. Stocker, Annu. Rev. Biophys. 43, 65 (2014).

[3] G. Li and J. X. Tang, Phys. Rev. Lett. 103, 078101 (2009).

[4] K. Drescher, J. Dunkel, L. H. Cisneros, S. Ganguly, and R. E. Goldstein, Proc. Natl. Acad. Sci. USA 108, 10940 (2011).

[5] J. Dunstan, G. Mino, E. Clement, and R. Soto, Phys. Fluids 24, 011901 (2012).

[6] S. E. Spagnolie and E. Lauga, J. Fluid. Mech. 700, 105 (2012).

[7] V. Kantsler, J. Dunkel, M. Polin, and R. E. Goldstein, Proc. Natl. Acad. Sci. USA 110, 1187 (2012).

[8] G. J. Li and A. M. Ardekani, Phys. Rev. E 90, 013010 (2014).

[9] S. E. Spagnolie, G. R. Moreno-Flores, D. Bartolo, and E. Lauga, Soft Matter 11, 3396 (2015).

[10] M. Contino, E. Lushi, I. Tuval, V. Kantsler, and M. Polin, Phys. Rev. Lett. 115, 258102 (2015).

[11] R. E. Goldstein, Annu. Rev. Fluid Mech. 47, 343 (2015).

[12] D. J. Smith, E. A. Gaffney, J. R. Blake, and J. C. KirkmanBrown, J. Fluid Mech. 621, 289 (2009).

[13] P. Denissenko, V. Kantsler, D. J. Smith, and J. C. KirkmanBrown, Proc. Natl. Acad. Sci. USA 109, 8007 (2012).

[14] M. Molaei, M. Barry, R. Stocker, and J. Sheng, Phys. Rev. Lett. 113, 068103 (2014).

[15] O. Sipos, K. Nagy, R. Di Leonardo, and P. Galajda, Phys. Rev. Lett. 114, 258104 (2015).

[16] E. Lauga, W. R. DiLuzio, G. M. Whitesides, and H. A. Stone, Biophys. J. 90, 400 (2006).

[17] R. Di Leonardo, D. Dell'Arciprete, L. Angelani, and V. Iebba, Phys. Rev. Lett. 106, 038101 (2011).
[18] D. Lopez and E. Lauga, Phys. Fluids 26, 071902 (2014).

[19] D. Takagi, J. Palacci, A. B. Braunschweig, M. J. Shelley, and J. Zhang, Soft Matter 10, 1784 (2014).

[20] A. T. Brown, I. D. Vladescu, A. Dawson, T. Vissers, J. SchwarzLinek, J. S. Lintuvuori, and W. C. K. Poon, Soft Matter 12, 131 (2016).

[21] S. Das, A. Garg, A. I. Campbell, J. Howse, A. Sen, D. Velegol, R. Golestanian, and S. J. Ebbens, Nat. Commun. 6, 8999 (2015).

[22] H. Wioland, F. G. Woodhouse, J. Dunkel, J. O. Kessler, and R. E. Goldstein, Phys. Rev. Lett. 110, 268102 (2013).

[23] E. Lushi, H. Wioland, and R. E. Goldstein, Proc. Natl. Acad. Sci. USA 111, 9733 (2014).

[24] H. Wioland, F. G. Woodhouse, J. Dunkel, and R. E. Goldstein, Nat. Phys. 12, 341 (2016).

[25] H. Wioland, E. Lushi, and R. E. Goldstein, New J. Phys. 18, 075002 (2016).

[26] K. Drescher, R. E. Goldstein, N. Michel, M. Polin, and I. Tuval, Phys. Rev. Lett. 105, 168101 (2010).

[27] J. S. Guasto, K. A. Johnson, and J. P. Gollub, Phys. Rev. Lett. 105, 168102 (2010).

[28] B. M. Friedrich and F. Julicher, Phys. Rev. Lett. 109, 138102 (2012).

[29] K. C. Leptos, K. Y. Wan, M. Polin, I. Tuval, A. I. Pesci, and R. E. Goldstein, Phys. Rev. Lett. 111, 158101 (2013).

[30] K. Y. Wan, K. C. Leptos, and R. E. Goldstein, J. R. Soc. Interface 11, 94 (2014).

[31] R. R. Bennett and R. Golestanian, Phys. Rev. Lett. 110, 148102 (2013).

[32] R. R. Bennett and R. Golestanian, J. R. Soc. Interface 12, 20141164 (2015). 
[33] L. Jibuti, L. Qi, C. Misbah, W. Zimmermann, S. Rafaï, and P. Peyla, Phys. Rev. E 90, 063019 (2014).

[34] J. P. Hernandez-Ortiz, C. G. Stoltz, and M. D. Graham, Phys. Rev. Lett. 95, 204501 (2005).

[35] J. P. Hernandez-Ortiz, P. T. Underhill, and M. D. Graham, J. Phys.: Condens. Matter 21, 204107 (2009).

[36] J. D. Weeks, D. Chandler, and H. C. Andersen, J. Chem. Phys. 54, 5237 (1971).

[37] R. Cortez, L. Fauci, and A. Medovikov, Phys. Fluids 17, 031504 (2005).

[38] J. Ainley, S. Durkin, R. Embid, P. Biondala, and R. Cortez, J. Comput. Phys. 227, 4600 (2008).

[39] See Supplemental Material at http://link.aps.org/supplemental/ 10.1103/PhysRevE.96.023102 for movies showing the swimmer scattering from a surface or escaping entrapment there by spinning about the main axis.
[40] M. Polin, I. Tuval, K. Drescher, J. P. Gollub, and R. E. Goldstein, Science 325, 487 (2009).

[41] H. H. Wensink, V. Kantsler, R. E. Goldstein, and J. Dunkel, Phys. Rev. E 89, 010302(R) (2014).

[42] R. Jeanneret, M. Contino, and M. Polin, Eur. Phys. J. Special Topics 225, 2141 (2016).

[43] K. Schaar, A. Zöttl, and H. Stark, Phys. Rev. Lett. 115, 038101 (2015).

[44] A. Wysocki, J. Elgeti, and G. Gompper, Phys. Rev. E 91, 050302(R) (2015).

[45] A. C. H. Tsang and E. Kanso, Phys. Rev. E 91, 043008 (2015).

[46] A. C. H. Tsang and E. Kanso, Phys. Rev. Lett. 116, 048101 (2016).

[47] T. Ostapenko, F. J. Schwarzendahl, T. J. Boddeker, C. T. Kreis, J. Cammann, M. G. Mazza, and O. Baumchen, arXiv:1608.00363. 